\title{
A BOUNDARY ELEMENT APPROACH TO BUCKLING OF LAMINATED PLATES SUBJECTED TO ARBITRARY IN-PLANE LOADING
}

\author{
Neeraj Cherukunnath ${ }^{*}$ and Stavros Syngellakis ${ }^{\dagger}$ \\ School of Engineering Sciences, \\ University of Southampton, Southampton SO17 1BJ, U.K.
}

\begin{abstract}
$\underline{\text { ABSTRACT }}$
Laminated plate buckling is analyzed by the boundary element method (BEM). Ignoring bendingstretching coupling, a solution is first sought for the membrane stresses due to arbitrary in plane loading. Using the stress function concept, it is shown that this problem is mathematically equivalent to the platebending problem. Based on this similarity, a new boundary element formulation is developed for the prediction of the pre-buckling membrane state of stress in an anisotropic plate. The integral equations for the buckling mode are then derived from a variational principle using the fundamental solution of the platebending problem. An irreducible domain integral depending on plate deflection rather than curvatures is numerically accounted for by adopting deflection modeling over the plate in addition to boundary modeling. Linear discontinuous boundary elements as well as domain cells are used along with special schemes for the approximation of jump term at corners. Analytical integration of singular integrals is performed over elements containing the source point. Thus a set of integral equations is transformed into an eigenvalue problem from which the critical load is evaluated. The reliability of the proposed analysis is established by comparing BEM predictions with solutions available from the literature or obtainable through a generalpurpose finite element program.
\end{abstract}

\section{INTRODUCTION}

Laminated composite panels are attractive structural elements in the aerospace and other industries due to their lightness and comparatively high strength and stiffness. Due to their slenderness however, buckling is one of the anticipated failure mechanism against which

\footnotetext{
${ }^{*}$ Postgraduate research student.

${ }^{\dagger}$ Senior Lecturer, Computational Engineering and Design Group.

Copyright $(2) 2001$ by the University of Southampton. Published by the American Institute of Aeronautics and Astronautics Inc. with permission.
}

panels need to be designed. Predictions of plate buckling and post-buckling require advanced modeling, particularly in cases of complex geometry, material behavior or loading. Numerical methods such as the finite element method have been commonly employed $^{1,2}$.

In this paper, a formulation based on the boundary element method (BEM) is presented. The only known attempts at a plate buckling analysis of laminated orthotropic plates using BEM were made by Shi and Bezine ${ }^{3}$ and $\mathrm{Shi}^{4}$, who adopted a direct approach based on their previous anisotropic plate bending analysis ${ }^{5}$. In these applications, constant boundary elements were used and results were obtained for uniformly loaded, simply supported or clamped rectangular plates.

A general buckling analysis should cater of any membrane stress distribution. For this reason, the emphasis here is on a new BEM formulation for the determination of the pre-buckling state using the stress function rather that the displacement components as field variable. The latter problem has already been solved by formulating integral equations in either real ${ }^{6}$ or complex ${ }^{7}$ domain. An alternative approach based on a modification of the generalised Hooke's law $^{8}$ allowed the use of Kelvin's fundamental solution for isotropic elastostatics. It seems however that the stress function concept has not been used for anisotropic BEM plane stress analysis.

It is shown that the boundary value problem for the stress function is almost identical to that governing plate flexure. Thus a BEM formulation for plane stress analysis can follow a very similar pattern to that of BEM solutions of the bending problem found in the literature ${ }^{5}$. The respective fundamental solutions have the same mathematical form but completely different physical interpretation and depend on different sets of anisotropic plate stiffness coefficients.

The boundary element formulation for the buckling analysis of a laminate plate is obtained in a similar manner to that adopted for isotropic plates 9 . The fundamental solution of the corresponding plate-

1

American Institute of Aeronautics and Astronautics 
bending problem is used. In contrast to previous formulations $\mathrm{s}^{3,4}$, the transformation of the integral equation of the problem is here performed in such a way that the deflection replaces the curvatures in the irreducible domain integrals.

The use of the same type of fundamental solution and its derivatives for the pre-buckling and buckling analyses is a convenient programming feature making possible the application of non-uniform edge loads at low computational cost. The numerical results obtained by the present method are compared with exact solutions and predictions from a general-purpose finite element package. The good agreement between these results indicates that the proposed scheme is a versatile analytical tool that can be reliably applied to the buckling analysis of laminate plates.

\section{GOVERNING EQUATIONS}

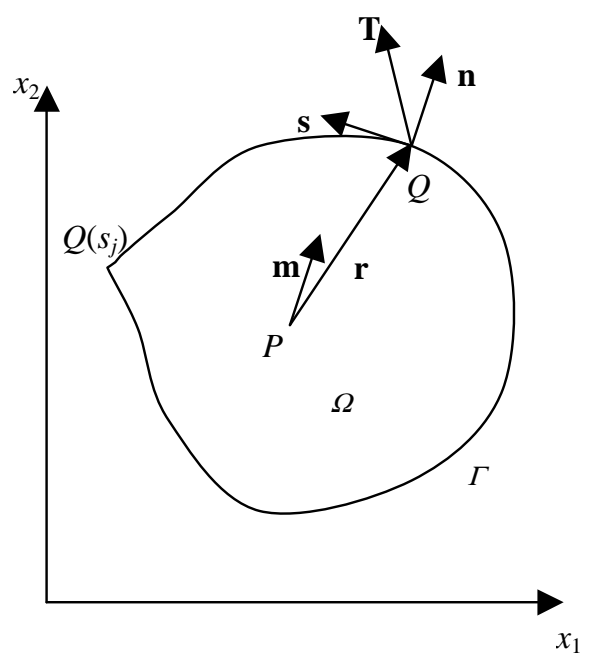

Fig. 1 Plate geometry notation

According to the classical lamination theory, the plate is assumed to be perfectly laminated consisting of an arbitrary number of discrete layers, each individual layer is homogeneous through its thickness and each layer is in a state of plane stress. The laminate is also assumed to deform according to Kirchhoff's assumption for the bending of thin plates. The laminated plates considered here are made by stacking orthotropic layers (or plies) symmetrically arranged about the middle reference surface. Bending-stretching coupling does not arise for these symmetrically laminated plates.
According to the above theory, the membrane stresses $N_{\alpha \beta}$ and bending moments $M_{\alpha \beta}$ are related to the curvatures $\kappa_{\alpha \beta}$ and mid-plane strains $\varepsilon_{\alpha \beta}$, respectively, by

$$
\begin{aligned}
N_{\alpha \beta} & =A_{\alpha \beta \gamma \delta} \varepsilon_{\gamma \delta} \\
M_{\alpha \beta} & =D_{\alpha \beta \gamma \delta} \kappa_{\gamma \delta}
\end{aligned}
$$

where $A_{\alpha \beta \gamma \delta}$ and $D_{\alpha \beta \gamma \delta}$ are, respectively, the extensional and flexural rigidities, the Greek indices take the values 1 and 2 and repeated indices mean summation over their range. If the plate is assumed loaded by in-plane body forces derivable from a potential function $\Phi$ and an un-factored edge load distribution $T_{\alpha}$, the problem is reduced to finding a stress function $F$ such that

$$
N_{\alpha \beta}=F,_{\kappa \kappa} \delta_{\alpha \beta}-F,_{\alpha \beta}+\Phi \delta_{\alpha \beta}
$$

where $\delta_{\alpha \beta}$ is the Kronecker delta and a comma followed by a lower index indicates differentiation with respect to the corresponding co-ordinate. In-plane equilibrium is identically satisfied by the stresses given by expressions (3), which also need to satisfy compatibility. This requirement leads to the differential equation

$$
A_{\alpha \beta \gamma \delta}^{\prime} F,_{\alpha \beta \gamma \delta}=f
$$

where

$$
\begin{gathered}
f=-A_{\alpha \beta \kappa \kappa}^{*}\left(\delta_{\alpha \beta} \nabla^{2} \Phi-\Phi,{ }_{\alpha \beta}\right) \\
A_{\alpha \beta \gamma \delta}^{\prime}=A_{\kappa \kappa \lambda \lambda}^{*} \delta_{\alpha \beta} \delta_{\gamma \delta}-A_{\alpha \beta \kappa \kappa}^{*} \delta_{\gamma \delta}-A_{\kappa \kappa \gamma \delta}^{*} \delta_{\alpha \beta}+A_{\alpha \beta \gamma \delta}^{*}
\end{gathered}
$$

and $A_{\alpha \beta \gamma \delta}^{*}$ is the inverse of the extensional rigidity tensor $A_{\alpha \beta \gamma \delta}$.

Using expressions (3) and referring to Fig. 1, it is possible to show that, at any point $Q\left(\bar{x}_{1}, \bar{x}_{2}\right)$ along the boundary,

$$
\begin{gathered}
F=\int_{O}^{Q}\left[\left(x_{1}-\bar{x}_{1}\right) T_{2}-\left(x_{2}-\bar{x}_{2}\right) T_{1}\right] \mathrm{d} \Gamma \\
\frac{\partial F}{\partial n}=-s_{1}(Q) \int_{O}^{Q} T_{1} \mathrm{~d} \Gamma-s_{2}(Q) \int_{O}^{Q} T_{2} \mathrm{~d} \Gamma
\end{gathered}
$$

where $O$ is an arbitrarily located origin and $\mathbf{s}\left(s_{1}, s_{2}\right)$ the unit tangent vector along $\Gamma$. According to Eq. (7), $F$ can be physically interpreted as the resultant moment about $Q$ of the traction over $O Q$. Similarly, Eq. (8) describes the normal derivative of $F$ as the component of the resultant traction over $O Q$ in the direction $-\mathbf{s}$ at $Q$.

\section{PRE-BUCKLING MEMBRANE STRESS ANALYSIS}

A BEM formulation for the stress function was developed noting that the corresponding problem

2

American Institute of Aeronautics and Astronautics 
described by Eqs. (4), (7) and (8) is mathematically very similar to that governing plate bending with the extensional compliances $A_{\alpha \beta \gamma \delta}^{\prime}$ replacing the flexural rigidities $D_{\alpha \beta \gamma \delta}$ and the function $f(\Phi)$ replacing lateral pressure. A reciprocity relation for the operator defined in Eq. (4) can be derived starting from the symmetric bi-linear form

$$
\Lambda\left(F, F^{*}\right)=\int_{\Omega} A_{\alpha \beta \gamma \delta}^{\prime} F,_{\alpha \beta} F^{*}{ }_{\gamma \delta} \mathrm{d} \Omega
$$

where $F$ and $F^{*}$ are two possible solutions, and $\Omega$ is the plate domain bounded by contour $\Gamma$, which is smooth apart from a finite number $K$ of corner points as shown in Fig. 1.

Repeated integration by parts and application of Green's theorem transforms Eq. (9) to:

$$
\begin{aligned}
\Lambda\left(F, F^{*}\right) & =\int_{\Omega} A_{\alpha \beta \gamma \delta}^{\prime} F,{ }_{\alpha \beta \gamma \delta} F^{*} \mathrm{~d} \Omega+\int_{\Gamma}\left[V(F) F^{*}\right. \\
& \left.-M_{n}(F) \theta_{n}\left(F^{*}\right)\right] \mathrm{d} \Gamma+\sum_{j=1}^{K} C_{j}(F) F_{j}^{*}=0
\end{aligned}
$$

where the operators $\theta_{n}(F), V(F)$, and $M_{n}(F)$ are given by

$$
\begin{gathered}
\theta_{n}(F)=\frac{\partial F}{\partial n} \\
V(F)=-A_{\alpha \beta \gamma \delta}^{\prime}\left[n_{\delta} F,_{\alpha \beta \gamma}+\frac{\partial}{\partial s}\left(n_{\gamma} s_{\delta} F,_{\alpha \beta}\right)\right] \\
M_{n}(F)=-A_{\alpha \beta \gamma \delta}^{\prime} n_{\gamma} n_{\delta} F,_{\alpha \beta}
\end{gathered}
$$

and the jump term $C_{j}$ represents the discontinuity of the expression

$$
M_{n s}(F)=-A_{\alpha \beta \gamma \delta}^{\prime} n_{\gamma} s_{\delta} F, \alpha \beta
$$

at the corners. An alternative right hand side to that appearing in Eq. (10) can be obtained by interchanging the roles of $F$ and $F^{*}$. Equating these equivalent forms of the functional $\Lambda\left(F, F^{*}\right)$ gives the reciprocity relation

$$
\begin{aligned}
\int_{\Omega} A_{\alpha \beta \gamma \delta}^{\prime}\left(F,_{\alpha \beta \gamma \delta} F^{*}-F^{*},{ }_{\alpha \beta \gamma \delta} F\right) \mathrm{d} \Omega \\
+I^{b}\left(F, F^{*}\right)+J\left(F, F^{*}\right)=0
\end{aligned}
$$

where

$$
\begin{aligned}
I^{b}\left(F, F^{*}\right)= & \int_{\Gamma}\left[V(F) F^{*}-M_{n}(F) \theta_{n}\left(F^{*}\right)\right. \\
& \left.+M_{n}\left(F^{*}\right) \theta_{n}(F)-V\left(F^{*}\right) F\right] \mathrm{d} \Gamma \\
J\left(F, F^{*}\right) & =\sum_{j=1}^{K}\left[C_{j}(F) F^{*}\left(s_{j}\right)-C_{j}\left(F^{*}\right) F\left(s_{j}\right)\right]
\end{aligned}
$$

The present formulation is based on Eq. (15) in which $F^{*}$ is chosen to be one of the singular fundamental solutions satisfying

$$
A_{\alpha \beta \gamma \delta}^{\prime} F_{i}^{*}{ }_{\alpha \beta \gamma \delta}=\delta_{i}(Q, P) ; i=1,2
$$

where $\delta_{1}(Q, P)$ is the Dirac-delta function , $\delta_{2}(Q, P)$ is the derivative of $\delta_{1}(Q, P)$ with respect to an arbitrary direction $\mathbf{m}$, while $P$ and $Q$ are the source and field points, respectively. These fundamental solutions are identical in form to those for the anisotropic platebending problem ${ }^{5}$. Their physical interpretation is of course different and should be related to unit potential and dipole sources referring to the forcing function $f$ defined by Eq. (5).

Introducing the fundamental solutions as weighting functions in Eq. (15) and ignoring body forces generates integral equations in the form

$$
-k F_{i}(P)+I^{b}\left(F, F_{i}^{*}\right)+J\left(F, F_{i}^{*}\right)=0 ; i=1,2
$$

where $k$ is equal to 1 or 0.5 depending on whether $P$ is in domain or on smooth portion of the boundary, respectively, and

$$
F_{1}=F, F_{2}=\partial F / \partial n
$$

The boundary values of $F$ and $\partial F / \partial n$ are computed using Eqs. (7) and (8) respectively. A boundary element solution of integral equation (19) can thus be generated that would provide the variation of $M_{n}(F)$ and $V(F)$ along the boundary. The membrane stresses at any point within the domain are then computed by differentiating twice the boundary integral equation for the stress function, that is, Eq. (19) with $k=1$ and $i=1$. These second partial derivatives are directly evaluated since they depend on known or already determined variables along the boundary.

\section{CRITICAL EQUILIBRIUM}

The membrane state of equilibrium determined through the procedure outlined in the previous section becomes unstable at a certain intensity of the factored edge load $\lambda T_{\alpha}$. Then a second stable equilibrium state exists, associated with a lateral deflection $w\left(x_{\alpha}\right)$ for a critical load factor $\lambda_{c}$. The variational equation governing the buckling mode $w\left(x_{\alpha}\right)$ has the form ${ }^{10}$

$$
\begin{aligned}
& \Pi(w, \delta w)=\int_{\Omega} D_{\alpha \beta \gamma \delta} w,_{\alpha \beta} \delta w,_{\gamma \delta} \mathrm{d} \Omega \\
& +\lambda_{c} \int_{\Omega} N_{\alpha \beta} w,{ }_{\alpha} \delta w,{ }_{\beta} \mathrm{d} \Omega=0
\end{aligned}
$$

where $\delta$ is the variation symbol.

Repeated integration by parts and application of Green's theorem transforms Eq. (20) to:

3 


$$
\begin{gathered}
\int_{\Omega}\left(D_{\alpha \beta \gamma \delta} w,_{\alpha \beta \gamma \delta}-\lambda_{c} N_{\alpha \beta} w,_{\alpha \beta}\right) \delta w \mathrm{~d} \Omega+\int_{\Gamma}\left[\left(V(w)+\lambda_{c} T_{\alpha} w,_{\alpha}\right) \delta w\right. \\
\left.-M_{n}(w) \delta \theta_{n}(w)\right] \mathrm{d} \Gamma+\sum_{j=1}^{K} C_{j}(w) \delta w_{j}=0
\end{gathered}
$$

where the normal slope $\theta_{n}(w)$, shear force $V(w)$ and bending moment $M_{n}(w)$ along the boundary are given by the same operators defined in Eqs. (11)-(13) but with the tensor $A_{\alpha \beta \gamma \delta}^{\prime}$ replaced by $D_{\alpha \beta \gamma \delta}$. Similarly, the corner forces $C_{j}(w)$ are equal to discontinuity jumps of the twisting moment $M_{n s}(w)$ as defined by Eq. (14).

The condition that Eq. (21) be satisfied for an arbitrary $\delta w$ yields the field equation

$$
D_{\alpha \beta \gamma \delta} w,_{\alpha \beta \gamma \delta}-\lambda_{c} N_{\alpha \beta} w,_{\alpha \beta}=0
$$

over the domain $\Omega$ and the boundary conditions,

$$
\begin{gathered}
\text { either } M_{n}=0 \text { or } \theta_{n}=\bar{\theta}(s) \\
\text { either } V+\lambda_{c} T_{\alpha} w,_{\alpha}=0 \text { or } w=\bar{w}(s)
\end{gathered}
$$

on $\Gamma$ and

$$
\text { either } C_{j}(s)=0 \text { or } w_{j}=\bar{w}\left(s_{j}\right) ; j=1, \ldots, K
$$

at the corners, where $\bar{\theta}(s)$ and $\bar{w}(s)$ are, respectively, prescribed values of the slope and deflection along the whole or part of the boundary. Thus Eq. (22) together with Eq. (4) are the uncoupled field equations governing buckling of symmetrically laminated plates subjected to in-plane forces. It is obvious that solving Eq. (4) is a prerequisite to the solution of the buckling problem governed by Eq. (22).

The boundary element formulation for the buckling mode is obtained in a similar manner to that applied for the stress function in the previous section. Initially, $\delta w$ in Eq. (20) is replaced by a weighting function $w^{*}$. Noting the symmetry of the bi-linear from $\Pi\left(w, w^{*}\right)$, a reciprocity relation

$$
\Pi\left(w, w^{*}\right)=\Pi\left(w^{*}, w\right)
$$

is deduced both sides of which can be transformed in a similar manner as that applied to the right hand side of Eq. (20). This leads to a new integral equation:

$$
\begin{aligned}
& \int_{\Omega} D_{\alpha \beta \gamma \delta}\left(w,_{\alpha \beta \gamma \delta} w^{*}-w^{*},{ }_{\alpha \beta \gamma \delta} w\right) d \Omega \\
&-\lambda_{c}\left[I^{d}\left(w, w^{*}\right)\right.\left.-I^{d}\left(w^{*}, w\right)\right]+\lambda_{c} I^{t}\left(w, w^{*}\right) \\
&+I^{b}\left(w, w^{*}\right)+J\left(w, w^{*}\right)=0
\end{aligned}
$$

where $I^{b}\left(w, w^{*}\right)$ and $J\left(w, w^{*}\right)$ are given by equations (14) and (15),

$$
I^{d}\left(w, w^{*}\right)=\int_{\Omega} N_{\alpha \beta} w,_{\alpha \beta} w^{*} d \Omega
$$

$$
I^{t}\left(w, w^{*}\right)=\int_{\Gamma} T_{\alpha}\left(w,_{\alpha} w^{*}-w^{*},{ }_{\alpha} w\right) d \Gamma
$$

The weighting function here used is replaced by the fundamental solutions of the plate bending problem satisfying

$$
D_{\alpha \beta \gamma \delta} w_{i}^{*},_{\alpha \beta \gamma \delta}=\delta_{i}(Q, P)
$$

they have therefore the same mathematical form as those defined through Eq. (18) but a completely different physical interpretation. More specifically, they are deflections of an infinite plate due to unit force and moment at the source point $P$.

Finally, similar to Eq. (15), integral equation (26) is transformed into

$$
\begin{aligned}
& -k w_{i}(P)+I^{b}\left(w, w_{i}^{*}\right)+\lambda_{c}\left[I^{t}\left(w, w_{i}^{*}\right)\right. \\
& \left.+I^{d}\left(w, w_{i}^{*}\right)\right]+J\left(w, w_{i}^{*}\right)=0 ; i=1,2
\end{aligned}
$$

where

$$
w_{1}=w, w_{2}=\partial w / \partial n
$$

Due to the presence of domain integral $I^{d}\left(w, w_{i}^{*}\right)$ depending on the unknown deflections, equation (30) is not a proper boundary integral equation. The boundary element methodology can still be applied to the present problem by introducing a simple domain deflection model instead of higher derivatives of deflection, complementing the conventional boundary modelling.

\section{INTERPOLATION MODELS}

The boundary integrals $I^{b}$ and $I^{t}$ in Eqs. (19) and (30) depend on four variables, either $F$ or $w, \theta_{n}, V$ and $M_{n} . V(F)$ and $M_{n}(F)$ are unknown quantities in Eq. (19) which need to be approximated over boundary elements. In Eq. (30), either $M_{n}$ or $\theta_{n}$, and $V$ or $w$ are the unknown quantities according to the boundary conditions (23)-(25). The linear discontinuous model has been adopted in the present analysis. This model represents more accurate variation of boundary variable and allows a direct modelling of its discontinuity at corners.

Interpolation functions for the discontinuous models are generated as polynomials in the natural co-ordinate $\xi$ with origin at the mid-point of the element. These functions should satisfy

$$
\phi_{i}\left(\xi_{j}\right)=\delta_{i j}
$$

where the range of indices depends on the order of approximation. In the case of linear interpolation the functions $\phi_{i}$ are given by

4 


$$
\phi_{1}=-\frac{\xi-\xi_{2}}{\xi_{2}-\xi_{1}}, \phi_{2}=\frac{\xi-\xi_{1}}{\xi_{2}-\xi_{1}}
$$

where $\xi_{1}$ and $\xi_{2}$ are the local co-ordinates of the two nodes. Since there are two independent unknowns per node, the total number of boundary unknowns will be $N_{b}=4 N_{e}$ where $N_{e}$ is the number of boundary elements.

If $Z$ denotes a boundary unknown, a typical boundary integral over an individual element $\Gamma_{e}$ would have the form

$$
I^{e}=\sum_{k=1}^{2} Z_{k} \int_{\Gamma_{e}} G \phi_{k} d \Gamma
$$

where $G$ would be the kernel paired with variable $Z$ in the integral equations (19) and (30). The integration over elements, which do not contain the source point are performed numerically using Gaussian quadarture. Analytical integration is carried out over elements containing the source point. With the source point identified with internal node " $i$ " of an element and a local co-ordinate $\mathrm{s}$ defined as shown in Fig. 2, the integrals over that element in the case of the linear interpolation model reduce to the form

$$
I^{e}=\frac{2 I_{1}^{e}}{L\left(\xi_{2}-\xi_{1}\right)}\left(Z_{2}-Z_{1}\right)+I_{0}^{e} Z_{i}
$$

where

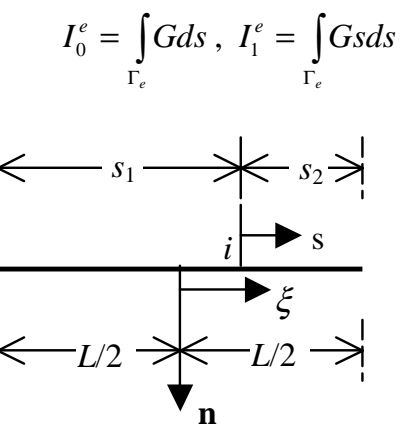

\section{Fig. 2 Boundary element co-ordinate for analytical integration}

The modelling of jump term in Eq. (19) at corners is achieved by deriving a relation between $C_{j}$ and certain boundary unknowns or their path derivatives. Eqs. (13) and (14) are transformed at the boundary relative to a local n-s frame of reference. This essentially involves the transformation of only the term $F,_{\alpha \beta}$ in eqs (13) and (14), which can be written

$$
\begin{gathered}
F,_{\alpha \beta}=n_{\alpha} n_{\beta} \frac{\partial^{2} F}{\partial n^{2}}+\left(n_{\alpha} s_{\beta}+s_{\alpha} n_{\beta}\right) \frac{\partial^{2} F}{\partial n \partial s} \\
+s_{\alpha} s_{\beta} \frac{\partial^{2} F}{\partial s^{2}}
\end{gathered}
$$

Substituting Eq. (36) into Eqs. (13) and (14) and eliminating $\partial^{2} F / \partial n^{2}$ between them, results in an expression for $C_{j}$ in terms of $M_{n}, \partial \theta_{n} / \partial s$ and $\partial^{2} F / \partial s^{2}$. Using the adopted boundary element model, an approximation can thus be deduced for $C_{j}$ appearing in the jump term over the boundary elements adjacent and on either side of a corner in terms of the nodal values of $M_{n}, \theta_{n}$ and $F$ within the elements. In a similar way, the jump term in Eq. (30) at corners is modelled in terms of the nodal values of $M_{n}, \theta_{n}$ and $w$ over the boundary elements adjacent and on either side of the corner.

The domain integral $I^{d}\left(w, w_{i}^{*}\right)$ in Eq. (30) is evaluated by modelling the deflection over the plate domain, which is discretized into $N_{c}$ triangular domain cells. Linear interpolation functions of the form

$$
\phi_{i}=\sum_{j=1}^{3} \alpha_{i j} \zeta_{j}
$$

were adopted where $\zeta_{j}, j=1,2,3$, are the familiar area co-ordinates. Thus the total number of internal nodes is $N_{d}=3 N_{c}$. With the three internal nodes placed along the medians halfway between the vertices and the cell centroid, the coefficients $\alpha_{i j}$ of the interpolation functions are given by

$$
\alpha_{i j}=\left\{\begin{array}{cc}
\frac{5}{3} & j=k \\
-\frac{1}{3} & j \neq k
\end{array}\right.
$$

\section{MATRIX FORMULATION}

The steps towards the numerical implementation of the developed formulation are described next. With the boundary divided into $N_{\mathrm{e}}$ linear discontinuous elements, integration of the terms appearing in integral equation (19), governing the stress function, is performed. This gives a consistent system of algebraic equations in the form

$$
\mathbf{H}_{0} \mathbf{Z}_{0}=\mathbf{G}_{0}
$$

where $\mathbf{X}_{0}$ is the array of the unknown nodal boundary values of $M_{n}(F)$ and $V(F), \mathbf{H}_{0}$ is a square coefficient matrix arising from the respective boundary integrals in $I^{b}\left(F, F_{i}^{*}\right)$, and $\mathbf{G}_{0}$ is a column matrix containing the boundary integrals depending on $F$ and $\partial F / \partial n$.

5 
Then a domain mesh is generated and the stresses $N_{\alpha \beta}$ are determined at all domain nodes. Boundary and domain models are substituted into Eq. (30), the source point placed at all boundary nodes and integrations are carried out over all elements leading to the following system of $N_{b}$ linear algebraic equations

$$
\left(\mathbf{H}^{b}+\lambda_{c} \mathbf{H}^{t}\right) \mathbf{Z}=\lambda_{c} \mathbf{H}^{d} \mathbf{W}
$$

where $\mathbf{Z}$ and $\mathbf{W}$ are the arrays containing the boundary and domain unknowns, respectively, the elements of matrix $\mathbf{H}^{b}$ result from integrals $I^{b}\left(w, w_{i}^{*}\right)$ and jump terms $J\left(w, w_{i}^{*}\right)$, while matrices $\mathbf{H}^{t}$ and $\mathbf{H}^{d}$ are the consequence of integrals $I^{t}\left(w, w_{i}^{*}\right)$ and $I^{d}\left(w, w_{i}^{*}\right)$, respectively. An additional system of $N_{d}$ equations,

$$
\left(\mathbf{D}^{b}+\lambda_{c} \mathbf{D}^{t}\right) \mathbf{Z}=\left(\mathbf{I}+\lambda_{c} \mathbf{D}^{d}\right) \mathbf{W}
$$

is obtained by applying Eq. (30) with $k=i=1$ and placing the source point at all domain nodes. Then, integrals $I^{b}\left(w, w_{1}^{*}\right), \quad I^{t}\left(w, w_{1}^{*}\right)$ and $I^{d}\left(w, w_{1}^{*}\right)$ would generate the elements of matrices $\mathbf{D}^{b}, \mathbf{D}^{t}$ and $\mathbf{D}^{d}$, respectively, with the jump terms contributing to $\mathbf{D}^{b}$.

The critical load factor is the smallest eigenvalue of the system of Eqs. (40) and (41) which can be written as a standard eigenvalue problem

$$
\left(\mathbf{A}-\lambda_{c} \mathbf{B}\right) \mathbf{X}=\mathbf{0}
$$

where

$$
\mathbf{A}=\left[\begin{array}{rr}
\mathbf{H}^{b} & \mathbf{0} \\
\mathbf{D}^{b} & -\mathbf{I}
\end{array}\right], \mathbf{B}=\left[\begin{array}{ll}
-\mathbf{H}^{t} & \mathbf{H}^{d} \\
-\mathbf{D}^{t} & \mathbf{D}^{d}
\end{array}\right], \mathbf{X}=\left\{\begin{array}{c}
\mathbf{Z} \\
\mathbf{W}
\end{array}\right\}
$$

Thus $\lambda_{c}^{-1}$ can be evaluated as the largest eigenvalue of matrix $\mathbf{A}^{-1} \mathbf{B}$ which can be directly obtained from

$$
\mathbf{A}^{-1} \mathbf{B}=\left[\begin{array}{cc}
-\hat{\mathbf{H}} b \mathbf{H}^{t} & \hat{\mathbf{H}} \mathbf{H}^{d} \\
-\mathbf{D}^{b} \hat{\mathbf{H}}^{b} \mathbf{H}^{t}+\mathbf{D}^{t} & \mathbf{D}^{b} \hat{\mathbf{H}}^{b} \mathbf{H}^{d}-\mathbf{D}^{d}
\end{array}\right]
$$

where $\hat{\mathbf{H}} b=\left(\mathbf{H}^{b}\right)^{-1}$. Thus the inversion of only the $N_{b} \times N_{b}$ matrix $\mathbf{H}^{b}$ is required while efficient routines yielding the largest eigenvalue of a matrix as well as the associated eigenvector are readily available. It is worth noting that boundary integral $I^{t}\left(w, w_{i}^{*}\right)$ vanishes under certain combinations of loading and support conditions. These include simply supported and clamped plates under edge shear anywhere on the boundary as well as compression on the clamped portion of the boundary. In such cases, both matrices $\mathbf{H}^{t}$ and $\mathbf{D}^{t}$ vanish and the eigenvalue problem (42) reduces further to

$$
\left[\left(\mathbf{D}^{b} \hat{\mathbf{H}} b \mathbf{H}^{d}-\mathbf{D}^{d}\right)-\lambda_{c}^{-1} \mathbf{I}\right] \mathbf{W}=\mathbf{0}
$$

Results can only be obtained in these special cases by implementing the above simpler formulation, which also considerably increases numerical efficiency.

\section{RESULTS AND DISCUSSION}

The BEM formulation was implemented through a suite of $\mathrm{C}$ codes. The plane stress analysis was first validated by applying it to a series of simple twodimensional problems. Stress results are here presented for the problem illustrated in Fig. 3(c). The orthotropic graphite/epoxy laminated panel shown was assumed to have the following extensional stiffness coefficients:

$$
\begin{aligned}
& A_{1111}=1.8181 \times 10^{9} \mathrm{~N} / \mathrm{m} \\
& A_{2222}=1.0346 \times 10^{8} \mathrm{~N} / \mathrm{m} \\
& A_{1122}=2.8969 \times 10^{7} \mathrm{~N} / \mathrm{m} \\
& A_{1212}=7.17 \times 10^{7} \mathrm{~N} / \mathrm{m}
\end{aligned}
$$

The aspect ratio $a / b$ of the plate was taken equal to 5 and a pressure $p=1 \mathrm{MPa}$ was applied on the top surface. The boundary was divided into a total of 120 elements (50 along each longer and 10 along each shorter side). The same plate was analyzed using ANSYS, a general-purpose finite element program ${ }^{11}$. A regular mesh of 400 8-node quadrilateral solid elements (Solid82) was adopted and symmetry was accounted for, that is, only one half of the plate was modeled.

The results from both analyses for the variation of $N_{11}$ and $N_{22}$ along the $x_{2}$-axis are shown in Fig. 4. Excellent agreement is noted. At most points, the difference between the two solutions is much less than $1 \%$. Greater discrepancies are observed near the edges probably due to the near singularity of the BEM kernels in these locations.

The buckling analysis was applied to simply supported and clamped square plates under various combinations of compression loads as shown in Fig. 3. Both uniform and non-uniform in-plane stress distributions were thus generated. The same plate material was assumed as that for the plane stress analysis. Its flexural rigidities were:

$$
\begin{aligned}
& D_{1111}=15151 \mathrm{Nm} \\
& D_{2222}=862.181 \mathrm{Nm} \\
& D_{1212}=597.5 \mathrm{Nm} \\
& D_{1122}=241.41 \mathrm{Nm}
\end{aligned}
$$

The critical value of a reference component of traction $T_{r c}$ is given in terms of the critical load factor $\lambda_{c}$ by

$$
T_{r c}=\lambda_{c} \frac{D_{3}}{a^{2}}
$$

where $D_{3}=D_{1122}+2 D_{1212}$ and $a$ the $x_{1}$-dimension of the plate as indicated in Fig. 3. The loading as well as the definition of $T_{r c}$ for each case analyzed can be seen in Table 1.

6 


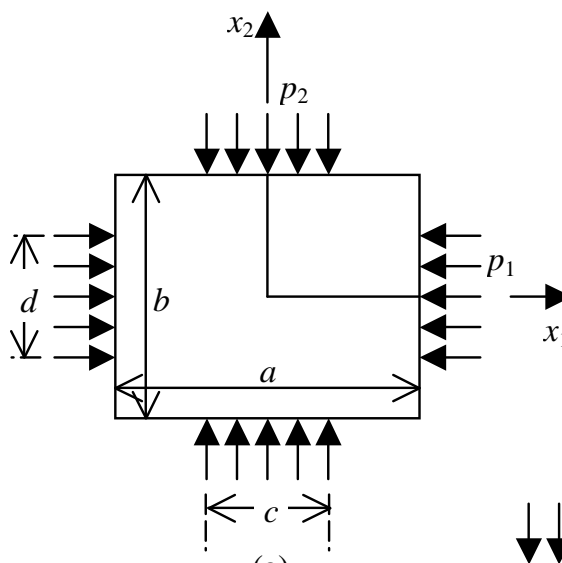

(a)

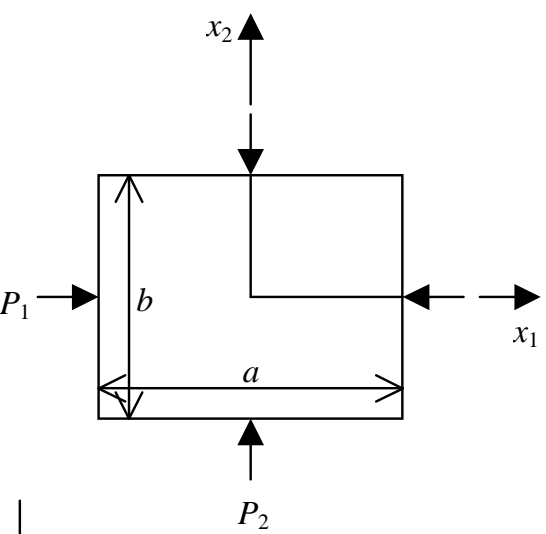

(b)

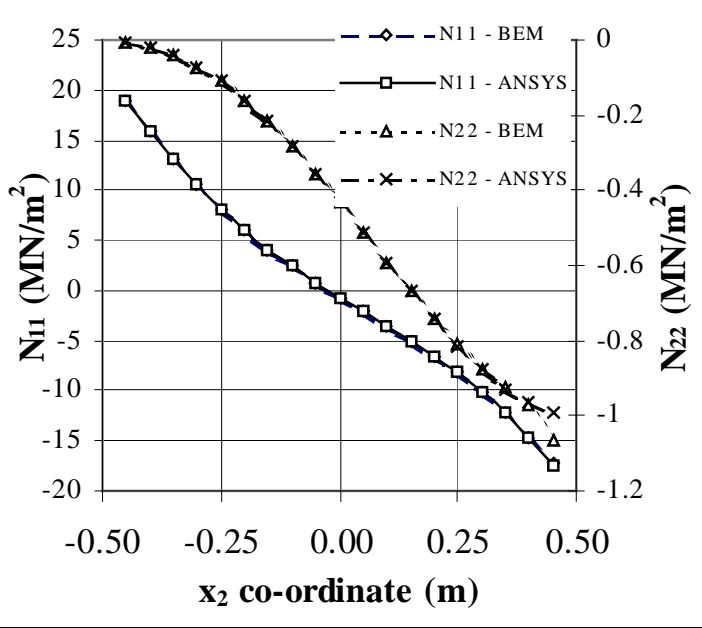

Fig. 4 Plane stress predictions for the problem shown in Fig. 3(c).

The results are also presented in Table 1 and can be compared to those obtained from ANSYS using comparable meshes. A total of 48 boundary elements and 50 domain cells are used for BEM analyses in comparison with 400 8-node finite shell elements $\left(\right.$ Shell $\left.93^{11}\right)$. The predicted critical loads from the two numerical methods for examples 1-3 are in very good agreement. Because of the uniformity of in-plane stresses, exact solutions are also available in these cases $^{12}$, which gave critical loads equal to 55.569, 129.766 , and 69.461, respectively. It is noted that the accuracy of the BEM predictions is the same or slightly higher than that of the FEM results.

Greater differences between BEM and FEM predictions are noted for examples 4-6 involving clamped plates. This may due to BEM satisfying more rigorously the edge constraints since the corresponding contour integrals vanish altogether while FEM allows some flexibility between nodes.

Even greater discrepancies are observed in the cases of applied point loads (examples 13-16) irrespective of boundary conditions. This is clearly due to the uniformity of both meshes, which do not quite capture the high stress concentrations in the neighbourhood of contact points. It was shown 
through numerical tests that increasing simply the number of boundary elements or refining their mesh in the neighbourhood of these points is not sufficient to improve the accuracy of the results. Domain mesh refinement is essential to the accuracy of both BEM and FEM analyses in the case of high stress concentrations.

Table 1: Critical load factor $\lambda_{c}$ for graphite/epoxy square plate.

\begin{tabular}{|c|c|c|c|c|c|c|}
\hline No. & Fig & $\mathrm{BC}$ & $T_{r c}$ & Loading & BEM & ANSYS \\
\hline 1 & (a) & $\mathrm{SS}$ & $p_{1}$ & $p_{1}=p_{2}, c=a, d=b$ & 55.520 & 55.449 \\
\hline 2 & (a) & $\mathrm{SS}$ & $p_{1}$ & $p_{2}=0, c=a, d=b$ & 130.089 & 129.448 \\
\hline 3 & (a) & $\mathrm{SS}$ & $p_{2}$ & $\mathrm{p}_{1}=0, c=a, d=b$ & 69.520 & 69.310 \\
\hline 4 & (a) & $\mathrm{CL}$ & $p_{1}$ & $\mathrm{p}_{1}=\mathrm{p}_{2}, c=a, d=b$ & 143.185 & 138.812 \\
\hline 5 & (a) & $\mathrm{CL}$ & $p_{1}$ & $\mathrm{p}_{2}=0, c=a, d=b$ & 468.200 & 458.777 \\
\hline 6 & (a) & $\mathrm{CL}$ & $p_{2}$ & $\mathrm{p}_{1}=0, c=a, d=b$ & 160.447 & 156.558 \\
\hline 7 & (a) & $\mathrm{SS}$ & $p_{1}$ & $\mathrm{p}_{1}=\mathrm{p}_{2}, c=a / 2, d=b / 2$ & 94.376 & 93.555 \\
\hline 8 & (a) & $\mathrm{SS}$ & $p_{1}$ & $\mathrm{p}_{2}=0, c=a / 2, d=b / 2$ & 160.705 & 157.971 \\
\hline 9 & (a) & $\mathrm{SS}$ & $p_{2}$ & $\mathrm{p}_{1}=0, c=a / 2, d=b / 2$ & 111.319 & 110.123 \\
\hline 10 & (a) & $\mathrm{CL}$ & $p_{1}$ & $\mathrm{p}_{1}=\mathrm{p}_{2}, c=a / 2, d=b / 2$ & 230.769 & 227.832 \\
\hline 11 & (a) & $\mathrm{CL}$ & $p_{1}$ & $\mathrm{p}_{2}=0, c=a / 2, d=b / 2$ & 527.909 & 517.715 \\
\hline 12 & (a) & $\mathrm{CL}$ & $p_{2}$ & $\mathrm{p}_{1}=0, c=a / 2, d=b / 2$ & 259.392 & 257.451 \\
\hline 13 & (b) & $\mathrm{SS}$ & $P_{1}$ & $P_{1}=P_{2}$ & 41.424 & 39.152 \\
\hline 14 & (b) & $\mathrm{SS}$ & $P_{1}$ & $P_{2}=0$ & 64.548 & 58.819 \\
\hline 15 & (b) & $\mathrm{CL}$ & $P_{1}$ & $P_{1}=P_{2}$ & 90.752 & 89.252 \\
\hline 16 & (b) & $\mathrm{CL}$ & $P_{1}$ & $P_{2}=0$ & 175.558 & 166.681 \\
\hline 17 & (c) & $\mathrm{SS}$ & $p$ & & 75.816 & 75.570 \\
\hline 18 & (c) & $\mathrm{CL}$ & $p$ & & 178.535 & 173.851 \\
\hline $\mathrm{B}: \mathrm{b}$ & & & & \\
\hline
\end{tabular}

BC: boundary conditions, SS: simply supported, CL: clamped

\section{CONCLUSIONS}

The proposed BEM plane stress analysis based on the stress function concept may lack the versatility of the displacement formulation since, in its present form, it is only applicable to a statically determinate configuration. It does however combine neatly with BEM formulations of the plate bending and buckling problems because of its mathematical similarity with these problems. It also provides very accurate predictions as was demonstrated through direct stress comparisons as well as indirectly from the critical load results. In certain cases, it would be possible to consider kinematic boundary conditions by replacing them by an assumed traction distribution and evoking SaintVenant's principle. Further research should lead to the generalisation of the method but at the cost of increasing its complexity.

Within the range of problems solved, the reliability of the buckling analysis can be considered satisfactory. Further convergence studies are required to establish better agreement between methods in the case of strongly non-uniform plane stress distributions. The method was tested on plates with very regular geometry and uniform boundary conditions. It is important to assess its reliability in cases of greater complexity.

With regard to the material model, the numerical algorithm and the developed program did account of general anisotropy although results for only orthotropic plates are presented here. Additional applications to plates with axial-shear coupling produced results in

8

American Institute of Aeronautics and Astronautics 
satisfactory agreement with those found in the literature $^{12}$.

There is considerable scope for further work. Extension-flexure coupling can be introduced and its effect on buckling loads assessed. Large deformation analysis leading to the prediction of the post-buckling behaviour is another possibility for expansion of the proposed formulation. This problem is of considerable design interest since it is linked more closely than the critical load to the strength limits of stiffened composite panels ${ }^{13}$.

\section{REFERENCES}

${ }^{1}$ Stein, M., "Postbuckling of Long Orthotropic Plates under Combined Loading," AIAA Journal, Vol. 23, Aug. 1985, pp. 1267-1272.

${ }^{2}$ Nemeth, P. M., "Importance of Anisotropy on Buckling of Compression-Loaded Symmetric Composite Plates," AIAA Journal, Vol. 24, Nov. 1986, pp. 1831-1835.

${ }^{3}$ Shi, G. and Bezine, G., "Buckling Analysis of Orthotropic Plates by Boundary Element Method," Mech. Res. Comm., Vol. 17, No. 1, 1990, pp. 1-7.

${ }^{4}$ Shi, G., "Flexural Vibration and Buckling Analysis of Orthotropic Plates by the Boundary Element Method," Int. J. Solids Structures, Vol. 26, No. 12, 1990, pp. 1351-1370.

${ }^{5}$ Shi, G. and Bezine, G., "A General Boundary Integral Formulation for the Anisotropic Plate Bending Problems," J. of Composite Materials, Vol. 22, Aug. 1988, pp. 694-716.

${ }^{6}$ Rizzo, F. J. and Shippy, D. J., "A Method for Stress Determination in Plane Anisotropic Elastic Bodies," $J$. of Composite Materials, Vol. 4, Jan. 1970, pp. 36-61.

${ }^{7}$ Lee, K. Y. U. J. and Mal, A. K., "A Boundary Element Method for Plane Anisotropic Elastic Media," J. Appl. Mechanics, Trans. ASME, Vol. 57, Sept. 1990, pp. 600-606.

${ }^{8}$ Perez, M. M. and Wrobel, L. C., "An Integral Equation Formulation for Anisotropic Elastostatics," $J$. Appl. Mechanics, Trans. ASME, Vol. 63, Dec. 1996, pp. 891-902.

${ }^{9}$ Syngellakis, S. and Elzein, A., "Plate Buckling Loads by the Boundary Element Method," Int. J. for Numerical Methods in Engineering, Vol. 37, No. 10, 1994, pp. 1763-1778.

${ }^{10}$ Budiansky, B., "Theory of Buckling and PostBuckling Behaviour of Elastic Structures," Advances in Applied Mechanics, ed. C. S. Yih, Academic Press: New York, Vol. 14, 1974, pp. 1-65.
${ }^{11}$ ANSYS 5.6, SAS IP, Inc., Canonsburg, PA, 1999.

${ }^{12}$ Whitney, J. M., Structural Analysis of Laminated Anisotropic Plates, Technomic Publishing, Ohio, 1987.

${ }^{13}$ Starnes, J. H., Knight, N. F. and Rouse, M., "Postbuckling Behavior of Selected Flat Stiffened Graphite-Epoxy Panels Loaded in Compression," AIAA Journal, Vol. 23, Aug. 1985, pp. 1236-1246. 